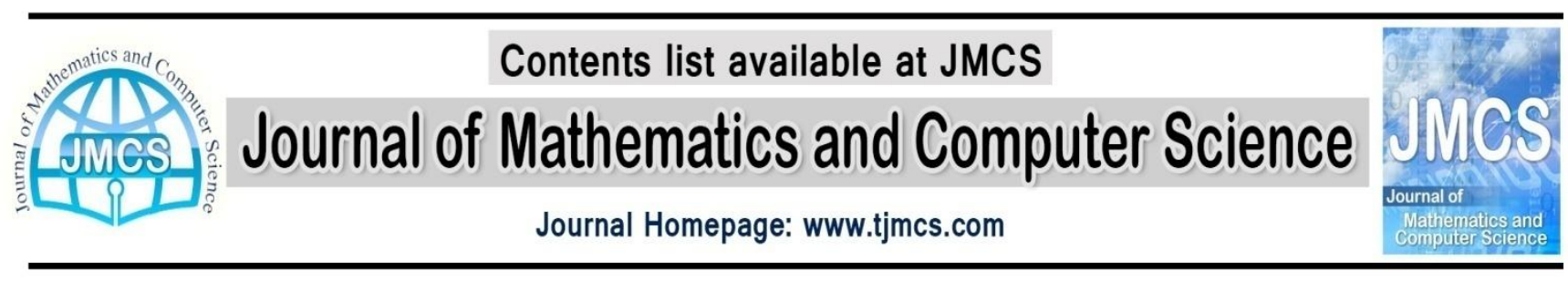

\title{
T-Rough Fuzzy Subgroups of Groups
}

\author{
Eshagh Hosseinpour \\ Department of Mathematics, Sari Branch, Islamic Azad University, Sari, Iran. \\ hosienpor_as51@yahoo.com
}

Article history:

Received July 2014

Accepted August 2014

Available online August 2014

\begin{abstract}
The rough set theory was introduced by Pawlak in 1982. It was proposed for presentation equivalence relations. But the concept of fuzzy set was introduced by Zadeh in 1965. In this paper,the concepts of the rough sets, $T$-rough sets, $T$-rough fuzzy sets, $T$-rough fuzzy subgroups, $T$-rough fuzzy ideals, and set-valued homomorphism of groups will be given. A necessery and sufficient condition for a fuzzy subgroup(ideal) and fuzzy prime ideal of a group under a set-valued homomorphism to be a $T$-rough fuzzy subgroup(ideal) and $T$-rough fuzzy prime ideal is stated. The purpose of this paper is to introduce and discuss the concept of $T$-rough fuzzy groups of groups that those have been proved in several papers. Also, we proved that intersection two fuzzy subgroups(ideals) of a set under a set-valued homomorphism is a $T$-rough fuzzy subgroup of other set.
\end{abstract}

Keywords: Approximation space, $T$-rough set, $T$-rough fuzzy set, Fuzzy subgroups, Fuzzy ideals, set-valued homomorphism.

\section{Introduction}

The notion of rough sets was introduced by Z.Pawlak in the year 1982 [24-25]. Rough set theory, a new mathematical approach to deal with inexact, uncertain or vague. Knowledge, has recently received wide attention on the research areas in both of the real-life applications and the theory itself. It has found practical applications in many areas such as knowledge discovery,machine learning, data analysis, approximate classification, conflict analysis, and so on [21-28]. Rough set theory is a mathematical framework for dealing with uncertainty and to some extent overlapping fuzzy set theory. The rough set theory approach is based on indiscernibility relations and approximations. The theory of rough set is an extension of set theory.

The concept of a fuzzy sets was introduced by Zadeh [32], and it is now a rigorous area of research with manifold applications ranging from engineering and computer science to medical diagnosis and social behavior studies. In particular, some researchers [12,15] applied the notion of fuzzy sets to ideals of a ring. The algebraic approach of rough sets was studied by some authors, for example, Q.M.Xiao and Z.L.Zhang [30] studied rough prime ideals and rough fuzzy prime ideals in semigroups. Based on the 
constructive method, extensive research has also been carried out to compare the theory of rough sets with other theories of uncertainty such as fuzzy sets and conditional events.

Since Rosenfeld [29], applied the notion of fuzzy sets to algebra and introduced the notion of fuzzy subgroups, since then many researchers are engaged in extending the concepts of abstract algebra to the broader framework of the fuzzy setting. In 1982, Liu [17] defined and studied fuzzy subrings and fuzzy ideals of a ring. Reader in $[4,9,10,11,14]$, will get some definitions and basic results about fuzzy algebras that their properties were carefully studied to a certain extent. Rough fuzzy sets and fuzzy rough sets are also studied by Nakamura [19], Nanda [20], Biswas [2,3], and by Banerjee and Pal [1]. Several research directions have been suggested on fuzzy rough sets and rough fuzzy sets.

In this paper, we introduce the concepts of the rough sets, T-rough sets, T-rough fuzz sets, Trough fuzzy subgroups(ideals), T-rough fuzzy prime ideal and set-valued homomorphism in a group and give some properties of such ideals and then extended some theorem in which have been proved in $[12,14]$.

\section{Preliminaries}

The following definitions and preliminaries are required in the sequel of our work and hence presented in brief. Some of them were in [21]. Suppose that $U$ is a non-empty set. A partition or classification of $U$ is a family $\theta$ of non-empty subsets of $U$ such that each element of $U$ is contained in exactly one element of $\theta$. Recall that an equivalence relation $\theta$ on a set $U$ is a reflexive, symmetric, and transitive binary relation on $U$. Eech partition $\theta$ induces an equivalence relation $\theta$ on $U$ by setting.

$$
x \theta y \Leftrightarrow x \text { and } y \text { are in the same class of } \theta .
$$

Conversely, each equivalence relation $\theta$ on $U$ induces a partition $\theta$ of $U$ whose classes have the form

$$
[x]_{\theta}=\{y \in U \mid x \theta y\} \text {. }
$$

Definition 2.1.[25]. A pair $(U, \theta)$ where $U \neq \phi$ and $\theta$ is an equivalence relation on $U$ is called an approximation space.

Definition 2.2.[25]. For an approximation space $(U, \theta)$ by a rough approximation in $(U, \theta)$ we mean a mapping Apr $: P(U) \rightarrow P(U) \times P(U)$ defined by for every $X \in P(U)$, $\operatorname{Apr}(X)=(\operatorname{Apr}(X), \overline{A p r}(X))$, where

$$
\underline{\operatorname{Apr}}(X)=\left\{x \in U \mid[x]_{\theta} \subseteq X\right\}, \overline{\operatorname{Apr}}(X)=\left\{x \in U \mid[x]_{\theta} \cap X \neq \phi\right\} .
$$

$\underline{\operatorname{Apr}}(X)$ is called a lower rough approximation of $X$ in $(U, \theta)$ whereas $\overline{\operatorname{Apr}}(X)$ is called a upper rough approximation of $X$ in $(U, \theta)$.

Definition 2.3. Given an approximation space $(U, \theta)$ a pair $(A, B)$ in $P(U) \times P(U)$ is called a rough set in $(U, \theta)$ if $(A, B)=(\underline{A p r}(X), \overline{A p r}(X))$ for some $X \in P(U)$.

Definition 2.4.[15]. Let $(U, \theta)$ be an approximation space. A subset fuzzy is a mapping $\mu$ from $U$ to $[0,1]$. If $x \in U$. We define

$$
\underline{\operatorname{Apr}}(\mu)(x)=\widehat{A}_{a \in[x]_{\theta}} \mu(a) \quad, \quad \overline{\operatorname{Apr}}(\mu)(x)={\underset{ }{\vee} \in[x]_{\theta}} \mu(a)
$$


They are called, respectively, the lower and the upper approximation of the fuzzy subset $\mu$. $\operatorname{Apr}(\mu)=(\underline{\operatorname{Apr}}(\mu), \overline{\operatorname{Apr}}(\mu))$ is called a rough fuzzy set respect to $\theta$ if $\underline{\operatorname{Apr}}(\mu) \neq \overline{\operatorname{Apr}}(\mu)$.

Definition 2.5.[15]. Let $\mu$ be a fuzzy subset of $G, t \in[0,1]$. Then the sets

$$
\mu_{t}=\{x \in G \mid \mu(x) \geq t\} ; \mu_{t}^{s}=\{x \in G \mid \mu(x)>t\}
$$

are called, respectively, t-levelest and t-strong levelest of the fuzzy set $\mu$.

Definition 2.6. 29 ]. A fuzzy subset $\mu$ of a group $G$ is called a fuzzy subgroup if, for all $x, y$ in $G$,

(1) $\mu(x y) \geq \mu(x) \wedge \mu(y)$;

(2) $\mu\left(x^{-1}\right) \geq \mu(x)$.

Definition 2.7.[29 ]. A fuzzy subset $\mu$ of a group $G$ is called a fuzzy ideal if, for all $x, y$ in $G$,

$$
\mu(x y) \geq \mu(x) \vee \mu(y)
$$

Definition 2.8.[15 ]. A fuzzy subset $\mu$ of a group $G$ is called a fuzzy prime ideal if, for all $x, y$ in $G$,

$$
\mu(x y)=\mu(x) \text { or } \mu(x y)=\mu(y)
$$

Definition 2.9. [ 15,18 ]. Let $\mu$ and $\lambda$ be two fuzzy subsets of $G$, then product $\mu \circ \lambda$ is defined by $(\mu \circ \lambda)(z)=\underset{z=x y}{\vee}(\mu(x) \wedge \lambda(y))$ for some $x, y \in G$.

\section{Set-valued homomorphism which induced by groups}

Definition 3.1. [ 5 ]. Let $X$ and $Y$ be two non-empty sets and $B \subseteq Y$. Let $T: X \rightarrow P^{*}(Y)$ be a setvalued mapping where $P^{*}(Y)$ denotes the set of all non-empty subsets of $Y$. The lower inverse and upper inverse of $B$ under $T$ are defined by

$$
T^{+}(B)=\{x \in X \mid T(x) \subseteq B\} ; T^{-1}(B)=\{x \in X \mid T(x) \cap B \neq \phi\} .
$$

Definition 3.2. [ 1 ]. Let $X$ and $Y$ be two non-empty sets and $B \subseteq Y$. Let $T: X \rightarrow P^{*}(Y)$ be a setvalued mapping where $P^{*}(Y)$ denotes the set of all non-empty subsets of $Y .\left(T^{+}(B), T^{-1}(B)\right)$ is called $T$-rough set of $G$.

Example 3.3. Let $(U, \theta)$ be an approximation space and $T: U \rightarrow P^{*}(U)$ be a set-valued mapping where $T(x)=[x]_{\theta}$, then for any $B \subseteq U, T^{+}(B)=\underline{A p r}(B)$ and $T^{-1}(B)=\overline{A p r}(B)$.

Definition 3.4. [ 29 ]. Let $G$ and $G^{\prime}$ be two groups and $T: G \rightarrow P^{*}\left(G^{\prime}\right)$ be a set-valued mapping. $T$ is called a set-valued homomorphism if for all $x, y \in G$,

(1) $\quad T(x y)=T(x) T(y)$;

(2) $\quad(T(x))^{-1}=\left\{a^{-1} \mid a \in T(x)\right\}=T\left(x^{-1}\right)$.

Remark 3.5. Example 3.3 is a set-valued homomorphism. So a group homomorphism is a special case of a set-valued homomorphism. Let $\theta$ be a complete congruence relation, i.e., $[x]_{\theta}[y]_{\theta}=[x y]_{\theta}$ for all $x, y \in G$. Define $T: G \rightarrow P^{*}(G)$ by $T(x)=[x]_{\theta}$ for all $x \in G$, then $T$ is a set-valued homomorphism. Further, by Example 3.3, the rough sets are $T$-rough sets. 
Definition 3.6.[12,13]. Let $G$ and $G^{\prime}$ be two groups and $T: G \rightarrow P^{*}\left(G^{\prime}\right)$ be a set-valued homomorphism. Let $\mu$ be a fuzzy subset of $G^{\prime}$. For every $x \in G$, we define

$$
T^{+}(\mu)(x)=\bigwedge_{a \in T(x)} \mu(a) ; T^{-1}(\mu)(x)={\underset{a \in T(x)}{\vee}}^{\vee} \mu(a) .
$$

$T^{+}(\mu)$ and $T^{-1}(\mu)$ are called, respectively, the $T$ - rough lower and the $T$-rough upper fuzzy subsets of $G$. If $T^{+}(\mu)$ and $T^{-1}(\mu)$ are fuzzy prime ideals, $\left(T^{+}(\mu), T^{-1}(\mu)\right)$ is said to be $T$ - rough fuzzy prime ideal of $G$.

Proposition 3.7. Let $G$ and $G^{\prime}$ be two groups and $T: G \rightarrow P^{*}\left(G^{\prime}\right)$ be a set-valued homomorphism. Let $\mu$ and $\lambda$ be two fuzzy subsets of $G^{\prime}$, then following hold:

(1) $T^{-1}(\mu \cup \lambda)=T^{-1}(\mu) \bigcup T^{-1}(\lambda)$;

(2) $T^{+}(\mu \bigcap \lambda)=T^{+}(\mu) \bigcap T^{+}(\lambda)$;

(3) $\mu \subseteq \lambda$ implies $T^{+}(\mu) \subseteq T^{+}(\lambda)$ and $T^{-1}(\mu) \subseteq T^{-1}(\lambda)$;

(4) $T^{-1}(\mu \cap \lambda) \subseteq T^{-1}(\mu) \cap T^{-1}(\lambda)$;

(5) $\quad T^{+}(\mu \cup \lambda) \supseteq T^{+}(\mu) \cup T^{+}(\lambda)$.

Proof. The proof is with using of definition.

Theorem 3.8. Let $G$ and $G^{\prime}$ be two groups and $T: G \rightarrow P^{*}\left(G^{\prime}\right)$ be a set-valued homomorphism. If $\mu$ is a fuzzy subgroup of $G^{\prime}$, then $T^{-1}(\mu)$ is a fuzzy subgroup of $G$.

Proof. Let $\mu$ is a fuzzy subgroup of $G^{\prime}$. Then we have for all $x, y \in G$,

$$
\begin{array}{r}
T^{-1}(\mu)(x y)=\underset{z \in T(x y)}{\vee} \mu(z) \geq \underset{a \in T(x), b \in T(y)}{\vee} \mu(a b) \\
\geq \underset{a \in T(x), b \in T(y)}{\vee}(\mu(a) \wedge \mu(b)) \\
=(\underset{a \in T(x)}{\vee} \mu(a)) \wedge(\underset{b \in T(y)}{\vee} \mu(b)) \\
=T^{-1}(\mu)(x) \wedge T^{-1}(\mu)(y) .
\end{array}
$$

Hence $T^{-1}(\mu)(x y) \geq T^{-1}(\mu)(x) \wedge T^{-1}(\mu)(y)$. And

$$
\begin{aligned}
T^{-1}(\mu)\left(x^{-1}\right) & =\underset{a^{-1} \in T\left(x^{-1}\right)}{\bigvee} \mu\left(a^{-1}\right) \geq \underset{a \in T(x)}{\bigvee} \mu(a) \\
& =T^{-1}(\mu)(x) .
\end{aligned}
$$

Therefore $T^{-1}(\mu)\left(x^{-1}\right) \geq T^{-1}(\mu)(x)$.

Theorem 3.9. Let $G$ and $G^{\prime}$ be two groups and $T: G \rightarrow P^{*}\left(G^{\prime}\right)$ be a set-valued homomorphism. If $\mu$ is a fuzzy subgroup of $G^{\prime}$, then $T^{+}(\mu)$ is a fuzzy subgroup of $G$.

Proof. Let $\mu$ is a fuzzy subgroup of $G^{\prime}$. Then we have for all $x, y \in G$, 


$$
\begin{array}{r}
T^{+}(\mu)(x y)=\underset{z \in T(x y)}{\vee} \mu(z) \geq \underset{a \in T(x), b \in T(y)}{\vee} \mu(a b) \\
\geq \underset{a \in T(x), b \in T(y)}{\vee}(\mu(a) \wedge \mu(b)) \\
=(\underset{a \in T(x)}{\vee} \mu(a)) \wedge(\underset{b \in T(y)}{\vee} \mu(b)) \\
=T^{+}(\mu)(x) \wedge T^{+}(\mu)(y) .
\end{array}
$$

Hence $T^{+}(\mu)(x y) \geq T^{+}(\mu)(x) \wedge T^{+}(\mu)(y)$. And

$$
\begin{aligned}
T^{+}(\mu)\left(x^{-1}\right)= & \underset{a^{-1} \in T\left(x^{-1}\right)}{\bigvee} \mu\left(a^{-1}\right) \geq \underset{a \in T(x)}{\bigvee} \mu(a) \\
& =T^{+}(\mu)(x) .
\end{aligned}
$$

Therefore $T^{+}(\mu)\left(x^{-1}\right) \geq T^{+}(\mu)(x)$.

Corollary 3.10. Let $G$ and $G^{\prime}$ be two groups and $T: G \rightarrow P^{*}\left(G^{\prime}\right)$ be a set-valued homomorphism. If $\mu$ is a fuzzy subgroup of $G^{\prime}$, then $\left(T^{+}(\mu), T^{-1}(\mu)\right)$ is a $T$ - rough fuzzy subgroup of $G$.

Theorem 3.11. Let $G$ and $G^{\prime}$ be two groups and $T: G \rightarrow P^{*}\left(G^{\prime}\right)$ be a set-valued homomorphism. If $\mu$ is a fuzzy ideal of $G^{\prime}$, then $\left(T^{+}(\mu), T^{-1}(\mu)\right)$ is a $T$-rough fuzzy ideal of $G$.

Proof. Let $\mu$ is a fuzzy ideal of $G^{\prime}$. Then we have for all $x, y \in G$,

$$
\begin{aligned}
T^{-1}(\mu)(x y)= & \underset{z \in T(x y)}{\vee} \mu(z) \geq \underset{a \in T(x), b \in T(y)}{\vee} \mu(a b) \\
& \geq \underset{a \in T(x), b \in T(y)}{\vee}(\mu(a) \vee \mu(b)) \\
& =(\underset{a \in T(x)}{\vee} \mu(a)) \vee(\underset{b \in T(y)}{\vee} \mu(b)) \\
& =T^{-1}(\mu)(x) \vee T^{-1}(\mu)(y) .
\end{aligned}
$$

Hence $T^{-1}(\mu)(x y) \geq T^{-1}(\mu)(x) \vee T^{-1}(\mu)(y)$. Also

$$
\begin{aligned}
& T^{+}(\mu)(x y)=\hat{z \in T(x y)}^{\mu(z) \geq} \hat{}_{a \in T(x), b \in T(y)} \mu(a b) \\
& \geq_{a \in T(x), b \in T(y)}(\mu(a) \vee \mu(b)) \\
& =(\underset{a \in T(x)}{\wedge} \mu(a)) \vee(\underset{b \in T(y)}{\wedge} \mu(b)) \\
& =T^{+}(\mu)(x) \vee T^{+}(\mu)(y) \text {. }
\end{aligned}
$$

Therefore $T^{+}(\mu)(x y) \geq T^{+}(\mu)(x) \vee T^{+}(\mu)(y)$

Proposition 3.12. Let $G$ and $G^{\prime}$ be two groups and $T: G \rightarrow P^{*}\left(G^{\prime}\right)$ be a set-valued homomorphism. Let $\mu$ and $\lambda$ be two fuzzy subsets of $G^{\prime}$, then following hold: 
(1) $T^{-1}(\mu \circ \lambda) \subseteq T^{-1}(\mu) \cap T^{-1}(\lambda)$;

(2) $T^{+}(\mu \circ \lambda) \subseteq T^{+}(\mu) \cap T^{+}(\lambda)$.

Proof.(1) For any $x, y, z \in G$, we have

$$
\begin{aligned}
T^{-1}(\mu \circ \lambda)(x)= & \underset{a \in T(x)}{\vee}(\mu \circ \lambda)(a)=\underset{a \in T(x)}{\vee}(\underset{a \in y z}{\vee}\{\min (\mu(y), \lambda(z))\}) \\
& \leq \underset{a \in T(x)}{\vee}(\underset{a \in y z}{\vee}\{\min (\mu(a), \lambda(a))\}) \\
& =\underset{a \in T(x)}{\vee}(\min \{\mu(a), \lambda(a)\})=\underset{a \in T(x)}{\vee}(\mu \cap \lambda)(a) \\
& =T^{-1}(\mu \cap \lambda)(x) .
\end{aligned}
$$

Therefore $T^{-1}(\mu \circ \lambda) \subseteq T^{-1}(\mu \cap \lambda) \subseteq T^{-1}(\mu) \cap T^{-1}(\lambda)$.

(2). The proof is similar to the proof (1).

Lemma 3.13. Let $\mu$ be a fuzzy subset of a group $G$. Then $\mu$ is a fuzzy subgroup of $G$ if and only if for all $t \in[0,1]$, if $\mu_{t}, \mu_{t}^{S} \neq \phi$, then $\mu_{t}$ and $\mu_{t}^{S}$ are fuzzy subgroups of $G$.

Proof. Assume $\mu$ is a fuzzy subgroup of $G$. We show that $x y \in \mu_{t}, x^{-1} \in \mu_{t}$ for all $x, y \in \mu_{t}$. Since $\mu$ is a fuzzy subgroup of $G$, we have $\mu(x y) \geq \mu(x) \wedge \mu(y) \geq t, \mu\left(x^{-1}\right) \geq \mu(x) \geq t$. Since $x, y \in \mu_{t}$, then $\mu(x) \geq t, \mu(y) \geq t$. It implies that $x y \in \mu_{t}, x^{-1} \in \mu_{t}$. Hence $\mu_{t}$ is a fuzzy subgroup of $G$.

Similarly, we can show $\mu_{t}^{s}$ is a fuzzy subgroup of $G$, too.

Conversely, let $\mu_{t}$ is a fuzzy subgroup of $G$ for all $t \in[0,1]$, and $\mu_{t} \neq \phi$. Assume $\mu(x) \wedge \mu(y)=t_{\circ}, \mu(x)=t_{\circ}$ for all $x, y \in G$. It implies $x, y \in \mu_{t_{\mathrm{o}}}$. Since $\mu_{t_{\mathrm{o}}}$ is a fuzzy subgroup of $G$, then $x y \in \mu_{t_{\mathrm{o}}}, \quad x^{-1} \in \mu_{t_{\circ}}$. Therefore $\mu(x y) \geq t_{\circ}=\mu(x) \wedge \mu(y), \mu\left(x^{-1}\right) \geq t_{\circ}=\mu(x)$. Hence $\mu$ is a fuzzy subgroup of $G$.

Lemma 3.14. Let $\mu$ be a fuzzy subset of a group $G$. Then $\mu$ is a fuzzy ideal of $G$ if and only if for all $t \in[0,1]$, if $\mu_{t}, \mu_{t}^{S} \neq \phi$, then $\mu_{t}$ and $\mu_{t}^{S}$ are fuzzy ideals of $G$.

Proof. The proof is similar to the proof lemma 3.13.

Lemma 3.15. Let $\mu$ be a fuzzy subset of a group $G$. Then $\mu$ is a fuzzy prime ideal of $G$ if and only if for all $t \in[0,1]$, if $\mu_{t}, \mu_{t}^{S} \neq \phi$, then $\mu_{t}$ and $\mu_{t}^{S}$ are fuzzy prime ideals of $G$.

Proof. Assume $\mu$ is a fuzzy prime ideal of $G$. Assume $\mu_{t} \neq \phi$. By lemma 3.14, $\mu_{t}$ is an ideal of $G$. Let $x, y \in G$, such that $x y \in \mu_{t}$. Since $\mu$ is a fuzzy prime ideal of $G$, then $\mu(x y)=\mu(x)$ or 
$\mu(x y)=\mu(y)$. It implies $x \in \mu_{t}$ or $y \in \mu_{t}$. Therefore $\mu_{t}$ is a prime ideal of $G$. Similarly, we can show $\mu_{t}^{S}$ is a prime ideal of $G$,too.

Conversely, assume for all $t \in[0,1]$, if $\mu_{t} \neq \phi$, then $\mu_{t}$ is a prime ideal of $L$. Let $x, y \in L, B y$ lemma 3.14, $\mu$ is a fuzzy ideal of $G$. It implies $\mu(x y) \geq \mu(x)$ and $\mu(x y) \geq \mu(y)$. Let $t=\mu(x y)$. Thus $x y \in \mu_{t}$. Since $\mu_{t}$ is a prime ideal of $G, x \in \mu_{t}$ or $y \in \mu_{t}$. It means that $\mu(x) \geq t=\mu(x y)$ or $\mu(y) \geq t=\mu(x y)$. Hence $\mu(x y)=\mu(x)$ or $\mu(x y)=\mu(y)$. Therefore $\mu$ is a fuzzy prime ideal of $G$. Lemma3.16 . Let $G$ and $G^{\prime}$ be two groups and $T: G \rightarrow P^{*}\left(G^{\prime}\right)$ be a set-valued homomorphism. If $\mu$ is a fuzzy ideal of $G^{\prime}$, then for any $t \in[0,1]$,

(1) $\quad\left(T^{+}(\mu)\right)_{t}=T^{+}\left(\mu_{t}\right)$;

(2) $\left(T^{-1}(\mu)\right)_{t}=T^{-1}\left(\mu_{t}\right)$;

(3) $\quad\left(T^{+}(\mu)\right)_{t}^{s}=T^{+}\left(\mu_{t}^{s}\right)$;

(4) $\left(T^{-1}(\mu)\right)_{t}^{s}=T^{-1}\left(\mu_{t}^{s}\right)$.

Proof. (1).

(2).

$$
\begin{aligned}
x \in\left(T^{+}(\mu)\right)_{t} & \Leftrightarrow T^{+}(\mu)(x) \geq t \Leftrightarrow \hat{a}_{a \in T(x)} \mu(a) \geq t \\
& \Leftrightarrow \forall a \in T(x), \mu(a) \geq t \\
& \Leftrightarrow T(x) \subseteq \mu_{t} \Leftrightarrow x \in T^{+}\left(\mu_{t}\right) .
\end{aligned}
$$

$$
\begin{aligned}
x \in\left(T^{-1}(\mu)\right)_{t} & \Leftrightarrow T^{-1}(\mu)(x) \geq t \Leftrightarrow \underset{a \in T(x)}{\vee} \mu(a) \geq t \\
& \Leftrightarrow \exists a \in T(x), \mu(a) \geq t \\
& \Leftrightarrow T(x) \cap \mu_{t} \neq \phi \Leftrightarrow x \in T^{-1}\left(\mu_{t}\right) .
\end{aligned}
$$

(3) and (4) are similar to (1) and (2).

Theorem 3.17. Let $G$ and $G^{\prime}$ be two groups and $T: G \rightarrow P^{*}\left(G^{\prime}\right)$ be a set-valued homomorphism. If $\mu$ is a fuzzy prime ideal of $G^{\prime}$, then $T^{+}(\mu)$ and $T^{-1}(\mu)$ are fuzzy prime ideals of $G$.

Proof. Let $\mu$ be a fuzzy prime ideal of $G^{\prime}$. By lemma 3.15, for all $t \in[0,1]$, if $\mu_{t} \neq \phi$, then $\mu_{t}$ is a prime ideal of $G$. By lemma 3.16 and 3.15, for all $t \in[0,1]$, if $\left(T^{+}(\mu)\right)_{t} \neq \phi,\left(T^{+}(\mu)\right)_{t}$ is a prime ideal of $G$. By lemma 3.15, $T^{+}(\mu)$ is a fuzzy prime ideal of $G$. Hence $\mu$ is a lower $T$-rough fuzzy prime ideal of $G$. Similarly, $\mu$ is a upper $T$-rough fuzzy prime ideal of $G$. Therefore $T^{+}(\mu)$ and $T^{-1}(\mu)$ are fuzzy prime ideals of $G$. 
Proposition 3.18. Let $G$ and $G^{\prime}$ be two groups and $T: G \rightarrow P^{*}\left(G^{\prime}\right)$ be a set-valued homomorphism. Let $\mu$ and $\lambda$ be two fuzzy subgroups( ideal) of $G^{\prime}$, then $\mu \bigcap \lambda$ is a upper $T$-rough fuzzy subgroup(ideal) of $G$.

Proof. Let $x, y \in G$, then

$$
\begin{aligned}
& \left.T^{-1}(\mu \bigcap \lambda)(x y)=\underset{z \in T(x y)}{\vee} \mu \bigcap \lambda\right)(z) \geq \underset{a \in T(x), b \in T(y)}{\vee}(\mu \bigcap \lambda)(a b) \\
& =\underset{a \in T(x), b \in T(y)}{\vee}(\min \{\mu(a b), \lambda(a b)\}) \\
& \geq \underset{a \in T(x), b \in T(y)}{\vee}(\min \{\min \{\mu(a), \mu(b)\}, \min \{\lambda(a), \lambda(b)\}\}) \\
& \geq \underset{a \in T(x), b \in T(y)}{\vee}(\min \{\min \{\mu(a), \lambda(a)\}, \min \{\mu(b), \lambda(b)\}\}) \\
& =\underset{a \in T(x), b \in T(y)}{\vee}(\min \{(\mu \cap \lambda)(a),(\mu \cap \lambda)(b)\}) \\
& =(\underset{a \in T(x)}{\vee}(\mu \cap \lambda)(a)) \wedge(\underset{b \in T(y)}{\vee}(\mu \cap \lambda)(b)) \\
& =T^{-1}(\mu \cap \lambda)(x) \wedge T^{-1}(\mu \cap \lambda)(y) .
\end{aligned}
$$

Therefore $T^{-1}(\mu \bigcap \lambda)(x y) \geq T^{-1}(\mu \bigcap \lambda)(x) \wedge T^{-1}(\mu \bigcap \lambda)(y)$.

Also

$$
\begin{aligned}
T^{-1}(\mu \cap \lambda)\left(x^{-1}\right) & =\underset{a^{-1} \in T\left(x^{-1}\right)}{\vee}(\mu \bigcap \lambda)\left(a^{-1}\right) \\
& \geq \underset{a \in T(x)}{\vee}(\mu \cap \lambda)(a)=T^{-1}(\mu \cap \lambda)(x) .
\end{aligned}
$$

Hence $\mu \bigcap \lambda$ is a upper $T$ - rough fuzzy subgroup of $G$.

Proposition 3.19. Let $G$ and $G^{\prime}$ be two groups and $T: G \rightarrow P^{*}\left(G^{\prime}\right)$ be a set-valued homomorphism. Let $\mu$ an $\lambda$ be two fuzzy subgroups( ideal) of $G^{\prime}$, then $\mu \bigcap \lambda$ is a lower $T$-rough fuzzy subgroup (ideal) of $G$.

Proof. The proof is similar to the proof proposition 3.18 .

\section{Conclusion}

Fuzzy set theory and rough set theory take into account two different aspects of uncertainty that can be encountered in real-world problems in many fields. Fuzzy sets deal with possibilities uncertainty, connected from ambiguity of information. The combination of fuzzy set and rough set lead to various models. This paper is intended to built up a connection between rough sets, fuzzy sets and groups theory. The notion of $T$ - rough fuzzy subgroups in a group is a generalization of the notion of fuzzy subgroups in a group. Also, using the concept of relation between rough fuzzy subgroups and level rough sets. In this paper, we substituted a universe set by a group, and introduced the set-valued homomorphism and $T$ rough fuzzy subgroup, and $T$-rough fuzzy ideals and $T$-rough fuzzy prime ideals in a group based on 
definitions in [12,13,16,30,31]. We generalized some ideas presented by Davvaz [5,6]. Further, we studied and investigated some their interesting properties of a set-valued homomorphism induced by a group homomorphism. We hope that this extended research many provide a powerful tool in approximate reasoning. Also, we believe, this paper offered here will turn out to be more useful in the theory and applications of rough sets and fuzzy sets.

\section{Acknowledgment}

The author is highly grateful to referees for their valuable comments and suggestion for improving the paper.

\section{References}

[1] M.Banerjee, and S.K. Pal, Roughness of a fuzzy set, Inform. Sci.93, (1996),235-246.

[2] R .Biswas, On rough sets and fuzzy rough sets, Bull. Pol.Acad. Sci. Math.42, (1994), 345-349.

[3] R. Biswas, On rough fuzzy sets, Bull. Pol. Acad. Sci. Math.42, (1994), 352-355.

[4] Z. Bonikowaski, Algebraic structures of rough sets, in: W.P. Ziarko (Ed.),Rough Sets, Fuzzy Sets and Knowledge Discovery, Springer-Verlag, Berlin, (1995), 242-247.

[5] B. Davvaz, A short note on algebraic T-rough sets ,Information Sciences , Vol.17, (2008) ,3247-3252.

[6] B. Davvaz, Roughness in rings, Inform. Sci. 164 ,(2004), 147-163.

[7] D.Dubois, H. Prade, Rough fuzzy sets and fuzzy rough sets, Int. J. General Syst. 17 (2-3),(1990), 191-209.

[8] D. Dubois, H. Prade, Two fold fuzzy sets and rough sets-some issues in knowledge representation, Fuzzy Sets Syst. 23, (1987), 3-18.

[9] E.Ranjbar-Yanehsari, M.Asghari-Larimi , union and intersection fuzzy subhypergroups, JMCS , Vol.5 , No.2, (2012)82-90.

[10] E. Hendukolaie, On fuzzy homomorphism between hypernear-rings , JMCS, Vol.2, No.4, (2011)702-716.

[11] E. Hendukolaie , M.Aliakbarnia.omran, Y.Nasabi, On fuzzy isomorphism theorems of $\Gamma$ - hypernear-rings, JMCS, Vol.7, (2013)80-88.

[12] S.B. Hosseini, N. Jafarzadeh, A. Gholami, T-rough Ideal and T-rough Fuzzy Ideal in a Semigrou , Advanced Materials Research, Vols.433-440,(2012), 4915-4919.

[13] S.B.Hosseini, N. Jafarzadeh, A. Gholami, Some Results on T-rough (prime, primary) Ideal and T-rough Fuzzy (prime, primary) Ideal on Commutative Rings ,Int.J.Contemp. Math Scince, Vol.7 , ( (2012), 337-350.

[14] T.Iwinski, Algebraic approach to rough sets, Bull. PolishAcad. Sci. Math.35, (1987), 673-683.

[15] O. Kazanci, B. Davvaz, On the structure of rough prime (primary) ideals and rough fuzzy prime (primary) ideals in commutative rings, Information Sciences, 178, (2008), 1343-1354.

[16] N.Kuroki, Rough ideals in semigroups, Inform. Sci. 100, (1997), 139-163.

[17] W.J. Liu, Fuzzy invariant subgroups and fuzzy ideals, Fuzzy Sets Syst. 8 ,1982) 133-139.

[18] J.N. Mordeson, M.S. Malik, Fuzzy Commutative Algebra, World Publishing, Singapore, 1998.

[19] A.Nakamura, Fuzzy rough sets, Note on Multiple-valued Logic in Japan,9 (8) ,(1988), 1-8.

[20] S. Nanda, Fuzzy rough sets, Fuzzy Sets and Systems, 45,(1992), 157-160.

[21] Z. Pawlak, Rough sets, Int. J. Inform. Comput. Sci. 11, (1982),341-356.

[22] Z. Pawlak, Rough sets- Theoretical Aspects of Reasoning about Data, Kluwer Academic Publishing, Dordrecht, 1991

[23] Z. Pawlak, Rough sets power set hierarchy, ICS PAS Rep.470, (1982).

[24] Z. Pawlak, Rough sets algebraic and topological approach, ICS PAS Rep. 482, (1982).

[25] Z. Pawlak, Rough sets and fuzzy sets , Fuzzy Sets and Systems. 17, (1985), 99-102.

[26] Z. Pawlak, Some remarks on rough sets, Bull. Pol. Acad.Tech. 33, (1985).

[27] Z. Pawlak, A. Skowron, Rough sets and Boolean reasoning, Information Sciences, 177, (2007), 41-73. 
[28] Z. Pawlak, A. Skowron, Rough sets: some extensions, Information Sciences, 177, (2007), 28-40.

[29] A. Rosenfeild, Fuzzy Groups, Journal of Mathematical Analysis and Application , 35, (1971) 512-517.

[30] Q.M. Xiao , Z.L. Zhang, Rough prime ideals and rough fuzzy prime ideals in semigroups, Information Sciences, 176, (2006), 725-733.

[31] S. Yamak, O. Kazanci , B. Davvaz, Generalized lower and upper approximations in a ring ,Information Sciences 180, (2010) 1759-1768.

[32] L.A. Zadeh, Fuzzy sets, Inform. Control 8, (1965) 338-353. 